Bundesgesundheitsbl 2020 63:251-253 https://doi.org/10.1007/s00103-020-03104-z Online publiziert: 13 . Februar 2020

(c) Der/die Autor(en) 2020

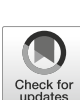

\author{
Wolfgang Ahrens ${ }^{1,2} \cdot$ Iris Pigeot $^{1,2} \cdot$ Tobias Pischon $^{3}$ \\ ' Leibniz-Institut für Präventionsforschung und Epidemiologie - BIPS, Bremen, Deutschland \\ ${ }^{2}$ Fachbereich Mathematik und Informatik, Universität Bremen, Bremen, Deutschland \\ ${ }^{3}$ Forschergruppe Molekulare Epidemiologie, Max-Delbrück-Centrum für Molekulare Medizin in der \\ Helmholtz-Gemeinschaft (MDC), Berlin, Deutschland
}

\title{
Was macht krank, was hält uns gesund?
}

Die Beantwortung dieser zentralen Fragen steht im Fokus der NAKO Gesundheitsstudie, der bislang größten populationsbasierten Kohortenstudie der in Deutschland lebenden Bevölkerung. Insgesamt werden 200.000 zufällig ausgewählte Einwohnerinnen und Einwohner im Alter von 20-69 Jahren aus verschiedenen Regionen Deutschlands medizinisch untersucht und hinsichtlich des Auftretens von häufigen chronischen Erkrankungen über mehrere Jahrzehnte weiterbeobachtet und nachuntersucht. Durch die Kombination umfangreicher Untersuchungs-, Bild- und Befragungsdaten mit zahlreichen biologischen Proben wird diese Langzeitstudie zukünftig neue Erkenntnisse zu den komplexen Ursachen und möglichen Vorzeichen vieler Volkskrankheiten liefern. Damit schafft sie die Grundlage für eine verbesserte Vorbeugung, Früherkennung und Behandlung dieser Erkrankungen.

Kohortenstudien sind das optimale Studiendesign, um solche Erkenntnisse $\mathrm{zu}$ gewinnen. Sie haben eine Tradition, die bis zum Anfang des 20. Jahrhunderts zurückreicht, wobei die dem heutigen Begriff entsprechenden, modernen Kohortenstudien erst nach Ende des Zweiten Weltkrieges initiiert wurden [1].Zu deren frühesten bekannten Vertretern gehören die British Doctors Study aus Großbritannien sowie die American Cancer Society Study und die Framingham Study aus den USA. Solche Kohortenstudien haben der wissenschaftlichen Forschung Erkenntnisse über Risikofaktoren geliefert, von denen viele heutzutage selbstverständliches Allgemeinwissen sind, wie Rauchen, erhöhter Blutdruck, erhöhter
Cholesterinspiegel oder ungesunde Ernährung [2].

In den letzten 10 Jahren sind in mehreren Ländern neue, sehr große Kohortenstudien aufgebaut worden, um dieser erfolgreichen Tradition zu folgen, wie beispielsweise die UK Biobank, die LifeGene-Studie in Schweden oder die Singapore Population Health Studies. Diese Entwicklung ist nicht zuletzt darauf zurückzuführen, dass insbesondere im Bereich der Labormethodik im letzten Jahrzehnt eine enorme Entwicklung stattgefunden hat, die es ermöglicht, in großen Studienkollektiven nicht nur einzelne Proteine, Metabolite oder genetische Polymorphismen zu untersuchen, sondern auch durch den Einsatz von Hochdurchsatzverfahren (Omics-Technologien) in relativ kurzer Zeit deren Gesamtheit im Organismus zu quantifizieren. Im Rahmen der sogenannten personalisierten Prävention liegt hier die Hoffnung, biologische Signaturen $\mathrm{zu}$ identifizieren, die eine verbesserte Vorhersage von Krankheiten erlauben. In Deutschland hat die „Kohortenforschung“ lange Zeit keine große Rolle gespielt - wie auch die Präventionsforschung insgesamt. Erst in den 1990er- und 2000er-Jahren wurden in Deutschland einige wenige mittelgroße Kohortenstudien zu spezifischen Fragestellungen [2] initiiert.

Die Publikation der ersten Ergebnisse zur Halbzeit der NAKO-Basiserhebung mit ca. 100.000 Teilnehmerinnen und Teilnehmern ist nicht nur ein Meilenstein für die Studie, sondern auch für die Zusammenarbeit epidemiologischer Forschungseinrichtungen in Deutsch- land. Die Planungen für die NAKO gehen zurück bis in das Jahr 2009. Damals war unklar, ob eine Studie dieser Größe über eine lange Laufzeit mit unterschiedlichsten Akteuren der universitären und außeruniversitären Forschung überhaupt erfolgreich auf den Weg gebracht werden kann. So besteht der NAKO e.V., der für die Durchführung der NAKO Gesundheitsstudie verantwortlich ist, heute aus 27 verschiedenen Mitgliedseinrichtungen und vereint Universitäten, Helmholtz-Zentren, LeibnizInstitute und andere Institutionen unter einem Dach. Die Studie wird mit öffentlichen Mitteln des Bundesministeriums für Bildung und Forschung, der Länder und der Helmholtz-Gemeinschaft sowie mit Eigenmitteln der beteiligten Institute gefördert. Bei einem Projekt dieser Größe und Heterogenität treffen unterschiedliche Meinungen aufeinander. Die NAKO Gesundheitsstudie hat diesen unterschiedlichen Meinungen Raum und Diskussionsforen gegeben, die zu einem gemeinsam abgestimmten Studienkonzept geführt haben. Maßgeblich für die gute Zusammenarbeit war sicherlich auch das gemeinsame Interesse an der Studie und ihren Ergebnissen. Hauptamtlich sind gegenwärtig ca. 460 Personen mit der direkten Studiendurchführung in den Mitgliedseinrichtungen befasst. Hinzu kommen das unterstützende Personal sowie die vielen Wissenschaftlerinnen und Wissenschaftler, die sich darüber hinaus in diese Studie einbringen.

Die Daten aus der NAKO Gesundheitsstudie werden pseudonymisiert und unter Wahrung des Datenschutzes 
für wissenschaftliche Forschungszwecke weltweit zugänglich gemacht. Die Entscheidung über die Datenherausgabe erfolgt auf Grundlage einer Nutzungsordnung, die auf der Homepage der NAKO Gesundheitsstudie einsehbar ist (www. nako.de). Dies betrifft selbstverständlich nur mehrfach pseudonymisierte Forschungsdaten. Personenidentifizierende Kontaktdaten sind hingegen nicht zugänglich und werden getrennt in einer zentralen Treuhandstelle gelagert. Die Beantragung von Forschungsdaten wird über ein Onlineportal erfolgen.

Die in diesem Heft präsentierten Ergebnisse der Basiserhebung erlauben eine Beschreibung der Grundgesamtheit der ersten ca. 100.000 Teilnehmenden der Studie, die seit 2014 untersucht wurden, und sind damit eine wichtige Voraussetzung für zukünftige wissenschaftliche Analysen. Diese Daten, die einen Einblick in die Zusammensetzung der Kohorte geben, die Untersuchungsmethoden genauer erläutern und zentrale gesundheitliche Charakteristika beschreiben, sollen in zwei Schwerpunktheften des Bundesgesundheitsblatts erstmals der Öffentlichkeit vorgestellt werden. Auch wenn diese Auswertung der Kohorte nicht den Anspruch erhebt, repräsentative Daten zur Prävalenz von Erkrankungen für Deutschland zu liefern, und einige der präsentierten Ergebnisse noch der Validierung bedürfen, so sind doch interessante Ergebnisse zur Verteilung und Korrelation wichtiger gesundheitlicher Parameter untereinander sowie aufschlussreiche Vergleiche mit den Ergebnissen anderer Studienpopulationen zu erwarten.

In diesem ersten Themenheft folgen nach einer Übersicht über die Basiserhebung der NAKO Gesundheitsstudie von Schipf und Co-Autoren, in der insbesondere das Untersuchungsprogramm und die Teilnahmequoten an den verschiedenen Modulen beschrieben werden, sieben Beiträge, die sich überwiegend der Verteilung wichtiger sozialer und medizinischer Charakteristika in der Studienpopulation und den Methoden zu ihrer Erfassung widmen.

So beschreibt der Beitrag von Dragano et al. die Verteilung der soziodemografischen Merkmale bei den
Teilnehmenden der NAKO Gesundheitsstudie und die hierfür eingesetzten Erhebungsinstrumente. Soziodemografische Faktoren wie Geschlecht, Alter und Bildung spielen eine zentrale Rolle bei der Prävention und bei der Entstehung von Erkrankungen, was in diesem Beitrag exemplarisch für einige Gesundheitsindikatoren wie dem Body-MassIndex aufgezeigt wird. Wiessner und Co-Autoren greifen diesen Aspekt für eine sehr heterogene und besonders vulnerable Gruppe, und zwar für Personen mit Migrationshintergrund, wieder auf und untersuchen u.a. an einem Beispiel den Zusammenhang zwischen Migrationshintergrund und der Nutzung von Gesundheitsangeboten.

Wie bereits das Beispiel des zweiten Beitrags zeigt, ist der Body-Mass-Index ein wichtiges Maß zur Einstufung von Personen als übergewichtig oder adipös. Er ist jedoch nur ein unzureichendes Maß für die Körperzusammensetzung und oft unzureichend, um das Risiko für Folgeerkrankungen der Adipositas $\mathrm{zu}$ untersuchen. Daher werden in dem Beitrag von Fischer et al. verbesserte Methoden zur anthropometrischen Messung der Körperzusammensetzung und -fettverteilung vorgestellt, die in der NAKO Gesundheitsstudie zum Einsatz kommen. Zudem werden erste deskriptive Ergebnisse zur Häufigkeit von Übergewicht und Adipositas in der Studienpopulation beschrieben.

Gerade in der Diskussion um Übergewicht und Adipositas wird körperliche Aktivität häufig als präventiver Faktor genannt. Um die präventive Wirkung von körperlicher Aktivität auf die Entstehung von Krankheiten besser beurteilen zu können, ist die genaue Erfassung dieses komplexen Verhaltens erforderlich. In der NAKO Gesundheitsstudie werden daher verschiedene komplementäre Methoden zur Erfassung körperlicher Aktivität eingesetzt, deren jeweiligen Anwendungsbereich Leitzmann und Kollegen herausstellen. Neben der körperlichen Aktivität ist auch die körperliche Fitness ein wesentlicher Prädiktor für Morbidität und Mortalität. Kluttig et al. stellen daher zum einen die in der NAKO Gesundheitsstudie eingesetzten Methoden zur Messung der körperlichen
Fitness, die aus den drei wesentlichen Komponenten kardiorespiratorische Fitness, Muskelkraft und Beweglichkeit besteht, vor und berichten zum anderen erste geschlechts- und altersspezifische Ergebnisse.

Die Beiträge in diesem Heft zeigen, wie die NAKO Gesundheitsstudie bundesweit nach einheitlichen Vorgaben und mit einer umfassenden Qualitätssicherung medizinische Charakteristika und potenzielle Einflussfaktoren auf die Gesundheit in der deutschen Bevölkerung untersucht. Viele dieser Faktoren und Merkmale sind zuvor nie bundesweit einheitlich erhoben worden, wie etwa der respiratorische Gesundheitszustand anhand der von Karrasch et al. beschriebenen Lungenfunktionsmessungen. Die Autorinnen und Autoren stellen hier u. a. erste Ergebnisse der Spirometrie und der Messung des exhalierten Stickstoffmonoxids vor und diskutieren die Ergebnisse, insbesondere in Bezug auf den Rauchstatus. Der letzte Beitrag widmet sich auch einem Thema, das in dieser Form noch nicht untersucht wurde, und zwar der Belästigung durch nächtlichen Verkehrslärm. Wolf und Co-Autoren zeigen dabei insbesondere Faktoren auf, die mit der nächtlichen Verkehrsbelästigung in $\mathrm{Zu}$ sammenhang stehen.

Insgesamt bieten diese Beiträge eine sehr gute Übersicht über die umfangreichen methodischen Werkzeuge, die in der NAKO Gesundheitsstudie zur Erfassung medizinischer Charakteristika und potenzieller Risikofaktoren eingesetzt werden. Darüber hinaus unterstreichen die in diesem Heft präsentierten Ergebnisse bereits die Bedeutung der NAKO Gesundheitsstudie für die deutsche $\mathrm{Ge}$ sundheitsforschung.

Wir möchten Sie an dieser Stelle auch auf das Folgeheft im April 2020 aufmerksam machen, das sich den Erhebungsmethoden und ersten Ergebnissen der NAKO Gesundheitsstudie mit Blick auf die großen Volkskrankheiten widmen wird, und wünschen Ihnen viel Freude bei der Lektüre beider Hefte,

Ihr Wolfgang Ahrens, Ihre Iris Pigeot und Ihr Tobias Pischon 


\section{Korrespondenzadresse}

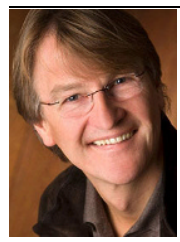

Prof. Dr. Wolfgang Ahrens

Leibniz-Institut für

Präventionsforschung und

Epidemiologie - BIPS

Achterstr. 30, 28359 Bremen,

Deutschland

ahrens@leibniz-bips.de

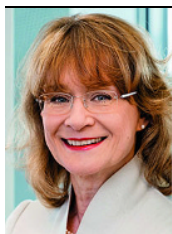

Prof. Dr. Iris Pigeot

Leibniz-Institut für

Präventionsforschung und

Epidemiologie - BIPS

Achterstr. 30, 28359 Bremen,

Deutschland

pigeot@leibniz-bips.de

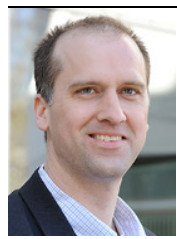

Prof. Dr. Tobias Pischon

Forschergruppe Molekulare

Epidemiologie, Max-

Delbrück-Centrum für

Molekulare Medizin in der

Helmholtz-Gemeinschaft

(MDC)

Robert-Rössle-Straße 10,

13125 Berlin, Deutschland

tobias.pischon@

mdc-berlin.de

Funding. Open Access funding provided by Projekt DEAL.

Interessenkonflikt. W. Ahrens, I. Pigeot und T. Pischon geben an, dass kein Interessenkonflikt besteht.

Open Access. Dieser Artikel wird unter der Creative Commons Namensnennung 4.0 International Lizenz veröffentlicht, welche die Nutzung, Vervielfältigung, Bearbeitung, Verbreitung und Wiedergabe in jeglichem Medium und Format erlaubt, sofern Sie den/die ursprünglichen Autor(en) und die Quelle ordnungsgemäß nennen, einen Link zur Creative Commons Lizenz beifügen und angeben, ob Änderungen vorgenommen wurden.

Die in diesem Artikel enthaltenen Bilder und sonstiges Drittmaterial unterliegen ebenfalls der genannten Creative Commons Lizenz, sofern sich aus der Abbildungslegende nichts anderes ergibt. Sofern das betreffende Material nicht unter der genannten Creative Commons Lizenz steht und die betreffende Handlung nicht nach gesetzlichen Vorschriften erlaubt ist, ist für die oben aufgeführten Weiterverwendungen des Materials die Einwilligung des jeweiligen Rechteinhabers einzuholen.

Weitere Details zur Lizenz entnehmen Sie bitte der Lizenzinformation auf http://creativecommons.org/ licenses/by/4.0/deed.de.

\section{Literatur}

1. Doll R (2001) Cohort studies: history of the method. I. Prospective cohort studies. Soz Praventivmed 46:75-86 (Erratum in: Soz Praventivmed 2002;47(2):90)

2. Ahrens W, Pigeot I (2012) Internationale Kohortenstudien. Bundesgesundheitsblatt Gesundheitsforschung Gesundheitsschutz 55:756-766 